$10.1590 / \mathrm{S} 1414-40772018000200010$

Este é um artigo publicado em acesso aberto sob uma licença Creative Commons https://creativecommons.org/licenses/by-nc/4.0/

\title{
Rankings acadêmicos na educação superior: tendências da literatura ibero-americana
}

\author{
Adolfo Ignacio Calderón ${ }^{1}$ \\ Carlos Marshal França ${ }^{2}$
}

Resumo: Apresenta-se o estado da questão sobre os rankings acadêmicos à luz da literatura ibero-americana. A partir de pesquisa bibliográfica, sistematizaram-se informações em duas categorias: estudos teóricos conceituais, referenciados nos paradigmas do conflito e do consenso, e estudos de referenciais empíricos, dividido em rankings globais, regionais e nacionais. Evidenciam-se, após 2010, predominância de estudos empíricos sobre a performatividade institucional e retração de estudos críticos ao ranqueamento.

Palavras-chave: Rankings acadêmicos. Educação superior. Rankings universitários.

\section{Academic rankings in higher education: trends of iberoamerica literature}

Abstract: The state of the question about academic rankings is presented in the context of Ibero-American literature. From a bibliographical research, information was systematized into two categories: conceptual theoretical studies, referenced in the paradigms of conflict and consensus, and empirical referential studies, divided into global, regional and national rankings. After 2010, there is a predominance of empirical studies on institutional performativity and retraction of critical studies to ranking.

Key words: Academic rankings. Higher education. University rankings. 


\section{Introdução}

Nas últimas décadas os rankings acadêmicos ${ }^{1}$ vêm ganhando destaque em todo o mundo, no espaço ibero-americano e também no Brasil. Como aponta Hazelkorn (2011), no mundo existe uma obsessão pelos rankings universitários, vinculados à maior prestação de contas e transparência, dentro de uma batalha pela excelência que, conforme a literatura internacional, geram diversas implicações na educação superior em termos de internacionalização e competição; governança e autonomia; e qualidade e produtividade (GONÇALVES; CALDERÓN, 2017). Apesar dos problemas existentes em termos metodológicos para avaliar instituições com missões e objetivos diferentes (ALTBACH, 2006), como bem enfatiza Marginson (2014), os rankings globais vieram para ficar. Nesse processo, emerge a disputa entre instituições e nações por possuir universidades que se incluam no exclusivo grupo das chamadas Universidades de Classe Mundial, uma condição de elite baseada no reconhecimento internacional decorrente do desempenho nos rankings, fato que possibilita avaliações comparativas em nível internacional (SALMI, 2009).

Os rankings acadêmicos internacionais de maior prestígio começaram a ser criados ao longo da década de 2000, começando com o ARWU - Academic Ranking of World Universities, criado em 2003, também conhecido como “classificação de Xangai”. Em 2004 foram criados o Webometrics Ranking of World Universities, produzido pelo Laboratorio de Cibermetría do Consejo Superior de Investigaciones Científicas (CISC) da Espanha e o World University Rankings, produzido pelo Times Higher Education Suplemente (THE), na época vinculado ao influente jornal inglês The Times (ORDORIKA; GOMEZ, 2010). Seguindo essa tendência internacional, conforme mapeamento realizado por Lourenço e Calderón (2015), os rankings acadêmicos também vêm proliferando no espaço ibero-americano, seja por meio de rankings regionais e nacionais.

No caso brasileiro, particularmente, a essa influência internacional, acima apontada, soma-se também a introdução de políticas públicas de regulação da educação superior que, já nos anos de 1995, suscitaram formas de ranqueamento de instituições de educação superior via introdução de exames de larga escala, de caráter obrigatório, aplicados junto a concluintes de

\footnotetext{
${ }^{1}$ Seguindo tendência de outros estudos sobre rankings na educação adota-se a expressão "rankings acadêmicos", pois não se limitam aos cursos de graduação, englobando a diversidade de atividades e funções relacionadas com o mundo universitário: cursos de graduação, cursos de pós-graduação, grupos de pesquisa científica, egressos e empregabilidade, transferência de conhecimento, inovação tecnológica, projeção internacional, entre outros.
} 
cursos superiores de graduação. A edição de um vasto aparato legal ao longo da segunda metade dos anos de 1990 e da primeira década dos anos 2000, parcialmente neutralizado em 2004 com a criação do Sistema Nacional de Avaliação da Educação Superior (SINAES), mas retomadas e potencializadas a partir de 2008, com a criação do Conceito Preliminar de Cursos (CPC) e o Índice Geral de Cursos (IGC), também contribuiu para o desenvolvimento de mecanismos de ranqueamento no país (CALDERÓN; POLTRONIERI; BORGES, 2011).

Apesar da expansão e da crescente importância, os rankings acadêmicos no Brasil têm sido estudados de modo incipiente, surgindo um emergente e inexplorado campo de pesquisas (CALDERÓN; PFISTER; FRANÇA, 2015). Esses fatos associados justificam o ineditismo do presente estudo, que tem por objetivo, por meio de um rigoroso levantamento e análise bibliográficos, traçar o atual estado da questão na literatura educacional ibero-americana sobre a temática dos rankings acadêmicos, identificando-se as tendências das pesquisas sobre esse tema nas últimas décadas. (NÓBREGA-THERRIEN; THERRIEN, 2004).

Os estudos selecionados para esta pesquisa foram captados de cinco bases de dados: Scielo - Scientific Eletronic Library Online; Redalyc - Red de Revistas Científicas de América Latina y el Caribe, España y Portugal; Portal de Períodos da Coordenação de Aperfeiçoamento de Pessoal de Nível Superior (CAPES) ${ }^{2}$; Educ@ - Publicações Online de Educação; e Directory of Open Access Journals (DOAJ). Deve-se destacar que em nenhuma dessas bases de dados foi identificado artigo com objetivo semelhante ao desenvolvido no presente trabalho focado especificamente na literatura ibero-americana, fato que revela sua originalidade.

Como corpus de análise, selecionaram-se artigos a partir da utilização dos descritores ranking e rankings, combinados com os adjetivos universitários ou acadêmicos. Esse procedimento permitiu identificar um total de 2.989 artigos (2.767 no Portal de Periódicos da CAPES; 157 no Redalyc; 41 no Scielo; 16 no Educ@; e 8 no DOAJ). Após profunda depuração das informações captadas nos motores de busca, que envolveu exame dos títulos e resumos desses artigos, e considerando-se apenas aqueles publicados nos idiomas português e espanhol, descartando-se artigos que tratavam dos rankings de forma periférica ou tangencial, identificaram-se cinquenta e sete artigos, publicados entre os anos de 1995 e 2016, que serviram como corpus de análise, os quais foram classificados, inicialmente, em duas categorias gerais:

\footnotetext{
${ }^{2}$ Biblioteca virtual, que disponibiliza mais de 38.000 periódicos científicos nacionais e internacionais e 120 bases referenciais de dados online, dentre elas, destacam-se a Scopus e a Web of Science como as mais completas e atualizadas para as ciências humanas.
} 
estudos teórico-conceituais e estudos de referenciais empíricos, cada uma delas com diversas vertentes como poderá ser observado ao longo deste artigo.

\section{Rankings acadêmicos: estudos teórico-conceituais}

$\mathrm{Na}$ categoria estudos teórico-conceituais englobaram-se artigos sobre a natureza dos rankings, suas finalidades, os papeis que desempenham na formulação de políticas públicas, seu impacto midiático, entre outros aspectos. Essa categoria foi desdobrada em duas vertentes, tendo como referência a distinção entre as perspectivas sociológicas dos paradigmas do conflito e do consenso (GOMES, 2005). A primeira vertente reúne artigos predominantemente críticos, que consideram os rankings ferramentas de avaliação das universidades a partir de parâmetros meritocráticos, quantitativista, alinhados às perspectivas neoliberais, sob tutela de organismos supranacionais como o Banco Mundial. A segunda vertente compreende os rankings, predominantemente, como veículos que permitem avaliar as universidades a partir de critérios objetivos, potencializando processo de melhoria dos produtos, serviços e processos universitários em um contexto de concorrência interinstitucional.

\section{a) Rankings acadêmicos na perspectiva do paradigma do conflito}

Nesta vertente, foram identificados dezessete estudos de referenciais teórico-conceituais enquadrados na perspectiva sociológica do paradigma do conflito, procedentes de autores vinculados a universidades de Brasil Argentina, Cuba, México, França, Chile.

Constatou-se um conjunto de artigos que tem como eixo principal o SINAES - Sistema Nacional de Avaliação da Educação Superior no Brasil. Barreyro (2006) ressalta que, ao desenvolver uma avaliação formativa, o SINAES representou uma mudança de paradigma na avaliação, então em curso no Brasil, presente a partir de meados dos anos noventa, quando se implantou no país o Exame Nacional de Cursos (ENC), que propiciou o ranqueamento das Instituições de Ensino Superior (IES). Rothen e Schulz (2007, p. 178) defendem que o conteúdo da Proposta do SINAES e sua legislação “constituiu um avanço em relação aos procedimentos de avaliação anterior", pois se cria um sistema integrado de avaliação, corrigindo parte das distorções do sistema anterior.

Estudo comparativo sobre experiências internacionais permitiu a Meneghel, Robl e Freitas da Silva (2006) afirmar que as políticas de avaliação e regulação na Argentina, na Espanha, em Portugal e na França revelam a existência de processos de avaliação externa às IES, sob a coordenação de órgãos governamentais, onde prevalece o princípio de uma avaliação 
formativa, sem que a mesma se confunda com mecanismos de regulação. Os autores também ressaltam os aspectos positivos do SINAES e concluem que a proposta do SINAES "vai ao encontro do que países com sistemas de avaliação da educação superior consolidados vêm realizando em termos de avaliação como referencial básico para a regulação” (MENEGHEL; ROBL; FREITAS DA SILVA, 2006, p. 104).

Zainko (2008) contextualiza esse movimento de mudança de paradigma ao efetuar uma análise histórica da avaliação da educação superior no Brasil, destacando que a avaliação de desempenho baseado na eficiência, de natureza regulatória, intensifica-se na década de 1990, particularmente nos governos de Fernando Collor e Fernando Henrique Cardoso, num contexto de clara redução do papel do Estado enquanto provedor de ensino superior, tendo como principal consequência a criação, em 1995, do ENC. A partir de 2004, segundo Zainko (2008), essa lógica é temporariamente suspensa com a instituição do SINAES, cujos documentos iniciais apontariam para uma concepção de avaliação emancipatória, processual, dinâmica. Três anos após a instituição dessa nova sistemática, com a criação do Conceito Provisório de Cursos (CPC) e do Índice Geral de Cursos (IGC), indicadores quantitativos relacionados aos cursos e instituições que permite ordená-los de modo classificatório, desencadeou-se novamente um processo de ranqueamento oficial no Brasil.

Barreyro (2008) também discute a introdução desses indicadores na legislação educacional brasileira, posicionando-se de maneira crítica e oposta à sua criação, à medida que os mesmos reforçariam tendências nocivas de ranqueamento das universidades. Esta deturpação dos princípios do SINAES é também apontada de forma enfática por autoras como Leite (2008) e Brito (2008). Para a primeira, os rankings construídos a partir do IGC e do CPC, e a sua subsequente publicidade via mídia esvaziam o papel dos outros mecanismos de avaliação institucional presentes no SINAES. Para a segunda autora, o ENADE, elaborado como uma sistemática de avaliação em larga escala, de caráter dinâmico, se modificou ao longo dos anos até se transformar num instrumento mais estático, muito semelhante ao extinto ENC, retomando-se um modelo que parecia ter sido ultrapassado com a instituição do SINAES. Por sua vez, Sguissardi (2008) busca discutir as relações existentes entre a regulação estatal e a cultura de avaliação da educação superior brasileira, tomando como ponto de partida e exemplificação a criação do CPC e do IGC, os quais, além de subsidiarem processos regulatórios, favorecem o processo de ranqueamento das instituições brasileiras.

A crítica a uma lógica mercantil aplicada ao ensino superior também se encontra presente nos artigos de autores como Charlot e Silva (2010); Chizzotti e Ponce (2010); e Moura, B. e Moura, L. (2013). Os primeiros discutem a questão dos rankings como consequência de 
importantes transformações ocorridas na universidade: internacionalização do ensino superior, mercantilização e crescente diferenciação entre as instituições. Os segundos, destacam que os rankings estariam diretamente associados aos conceitos de mercado, regulação, nova gestão pública, racionalidade, performance e rendimento, e sua crescente valorização pelos veículos de mídia contribui com o fenômeno de mercadorização da educação superior. Moura, B. e Moura, L. (2013), por sua vez, enfatizam o efeito cada vez maior dos rankings no processo de transformação da missão das IES, por meio da inclusão de estratégias corporativas típicas das empresas mercantis; do risco de homogeneização de desempenho; e de distorções na aplicação dos recursos para investimento.

Não são apenas autores brasileiros que vem desenvolvendo estudos teóricos sobre os rankings a partir da abordagem do conflito. Ordorika Sacristán (2006) discute o fenômeno dos rankings internacionais no contexto do processo de globalização e identifica nos rankings tentativa de construção de um discurso de legitimação ideológica, ao difundir um modelo ideal de universidade de excelência a ser perseguido e valorizado universalmente, reforçando alinhamento e hierarquização, elementos nocivos ao próprio conceito de universidade e aos interesses nacionais de países periféricos.

Lopez Segrera $(2008 ; 2009$; 2016) sintetiza tendências e desafios da educação superior na América Latina e no Caribe ao longo das últimas duas décadas, decorrentes da globalização e das orientações do Banco Mundial, dentre elas: o surgimento e fortalecimento dos rankings. Para esse autor as contrarreformas orientadas a satisfazer as demandas da política do Banco mundial não são as reformas necessárias para uma universidade pública nacional.

Barsky (2012) discute a repercussão da divulgação dos resultados dos rankings internacionais na Argentina e seu impacto nos processos de avaliação da qualidade universitária argentina. Após apresentar e discutir o contexto em que essas tabelas de classificação surgem; o impacto midiático que promovem; e as principais limitações metodológicas presentes em todos eles, concluem destacando seu papel muito mais midiático do que científico e suas limitações e impropriedades para uma efetiva avaliação do sistema universitário argentino.

Reyes (2016) efetua uma análise crítica sobre os rankings, tanto em relação à sua construção como às suas formas de divulgação. Com base nos conceitos sociológicos de performatividade e da noção de fact-totem, e a partir de entrevistas semiestruturadas com reitores e especialistas em qualidade universitária chilenos, a autora analisa os rankings universitários procurando explicitar a ideia de qualidade existente por trás dos mesmos e como essa ideia influencia no funcionamento das instituições. Dentre as conclusões encontradas, destaca-se que os rankings contribuem para a instalação de uma determinada ideia de qualidade, 
em relação à qual as universidades respondem sistematicamente por meio de condutas que acabam por atender exatamente ao modelo de qualidade esperado.

\section{b) Rankings acadêmicos na perspectiva do paradigma do consenso.}

Nesta vertente, foram identificados onze estudos de referenciais teórico-conceituais na perspectiva sociológica do paradigma do consenso, procedente de autores vinculados a universidades de Espanha, Brasil, México e Honduras.

Rodríguez Sabiote e Gutiérrez Pérez (2003) discutem o processo de avaliação da qualidade vivenciado pelas universidades espanholas desde o início dos anos de 1990 evidenciando problemas comuns nos processos de avaliação universitária em geral, e na construção de rankings em particular, principalmente de natureza técnica e política na escolha e construção dos indicadores. Embora reconheça a utilidade jornalística dos rankings, o autor questiona metodologicamente a construção dos rankings e aponta ser arriscado utiliza-los como parâmetros para as decisões acerca do aporte ou não de recursos e investimentos em determinadas instituições. Diante essas constatações, elenca propostas de melhoria no estabelecimento de critérios técnicos de seleção dos indicadores de medida.

Enquanto Rodríguez Sabiote e Gutiérrez Perez (2003) efetuam uma análise do lugar que os rankings ocupam no espaço europeu, Calderón, Poltronieri e Borges (2011) e Calderón e Lourenço (2014) desenvolvem estudos teóricos que procuram compreender como os rankings vêm se transformando, no Brasil, de políticas de governo em políticas de Estado. A hipótese do primeiro desses trabalhos é a de que, mesmo em governos diversos, representativos de projetos políticos opostos, os rankings vêm ganhando legitimidade técnica no interior do aparelho do Estado brasileiro, e vem construindo consensos enquanto estratégias que permitem estimular uma concorrência interinstitucional positiva entre as universidades, que pode contribuir para a melhoria da qualidade dos serviços de educação superior do Brasil. O segundo trabalho, por sua vez, aborda a trajetória dos primeiros rankings públicos e privados no Brasil, associandoas às mudanças relacionadas ao papel do Estado (de um Estado provedor para um Estado avaliador), num cenário em que se reconfiguram os instrumentos de avaliação destinados a mensurar a qualidade das universidades.

A essa literatura somam-se Anzaldo (2004) e Calderon Padilla (2011). O primeiro autor se ocupa da análise da realidade mexicana a partir dos resultados das universidades desse país no ARWU, destacando o quanto as universidades mexicanas encontram-se distantes do perfil de universidade vocacionada para a pesquisa. Longe de criticar a metodologia utilizada para a construção desse ranking, Anzaldo (2004) assume uma posição de defesa dos rankings em 
geral, à medida que eles podem ser utilizados para aferir-se a qualidade das universidades, atividade necessária para garantir que elas cumpram suas funções nas sociedades modernas. Também tomando como referência resultados em rankings internacionais, Calderon Padilla (2011) analisa a realidade da educação superior em Honduras a partir do desempenho da Universidad Nacional Autonoma de Honduras no Ranking Iberoamericano de Universidades produzido pela SCImago Institutions Rankings (SIR), defende que os indicadores adotados nos rankings devem servir aos tomadores de decisões para impulsar políticas que posicionem melhor as universidades hondurenhas entre as universidades de ibero-americanas.

Com o propósito de apresentar uma breve descrição sobre as principais características dos rankings de maior prestígio no mundo, Seoane (2009) apresenta as características gerais dos principais componentes utilizados na construção de nove rankings universitários da Europa, Estados Unidos, Austrália e Canadá. Segundo esse autor, os rankings respondem às demandas dos consumidores de serviços de educação superior, à medida que revelam o posicionamento relativo de uma universidade em relação às demais, podendo contribuir para definir os critérios a partir dos quais se atribui o conceito de qualidade da educação superior.

A relevância dos rankings enquanto instrumentos de avaliação da reputação e da qualidade das Universidades e sua influência numa eventual relação de consumo estabelecida entre estudantes e universidades são estudados por Telles Scaglione e Pitpar Goldchleger (2016) em trabalho dedicado a compreender o comportamento dos jovens na escolha de uma universidade.

As principais conclusões de Seoane (2009) e Calderon Padilla (2011), isto é, o potencial dos rankings para impulsionar melhorias de qualidade são compartilhadas por Villaseñor Becerra, Moreno Arellano e Flores Orozco (2015), que chegam a afirmar que os rankings devem ser vistos de maneira pragmática, independente das muitas críticas que possam sofrer quanto à suas deficiências técnicas ou metodológicas.

Questões técnicas também fazem parte das preocupações de Seoane e Álvarez (2010) que destacam a importância da adequada construção de indicadores como elemento constitutivo dos instrumentos de avaliação, tendo em consideração a própria dificuldade da construção de consensos sobre o que seja avaliação e a necessidade de gerar mecanismos de avaliação válidos e reconhecidos pelos diversos públicos.

Martins (2015) discute as recíprocas relações existentes entre o chamado processo de globalização e as mudanças pelas quais vem passando a educação superior. Num contexto marcado pelos fenômenos da globalização, da desterritorialização, da mobilidade acadêmica transnacional, da construção das world class universities, a emergência dos rankings 
internacionais é abordada como um dos produtos desse processo de formação de um sistema transnacional de ensino superior.

Calderón, Pfister e França (2015), com base em um estudo sobre o estado da questão sobre rankings na educação superior brasileira, postulam a hipótese de que, embora ainda incipiente, parece haver a emergência de um campo de estudos específico destinado ao fenômeno dos rankings a partir do início dos anos 2000.

\section{Rankings acadêmicos: estudos referenciais empíricos}

Nesta categoria englobam-se artigos que descrevem ou analisam objetivamente os rankings acadêmicos existentes ou eventualmente propostos, bem como enfocam aspectos de natureza metodológica de um determinado ranking em particular ou entre diferentes rankings. Esta categoria se desdobra, por sua vez, em três vertentes: estudos a respeito de rankings globais; regionais; e nacionais.

\section{a) A respeito de rankings globais}

Foram identificados nove estudos sobre rankings internacionais na literatura acadêmica educacional ibero-americana, procedente de autores vinculados a universidades de Brasil, Espanha, México, Estados Unidos, França, Portugal e Venezuela. Ordorika e Gomez (2010) destacam o papel dos rankings, de modo geral, nas dinâmicas de avaliação das instituições de educação superior nos últimos anos. Analisam o ranking editado e publicado pelo Times Higher Education, enfatizando os antecedentes históricos, os parâmetros metodológicos a partir dos quais ele é construído, a evolução de seus resultados no período 2004-2009 e a posição das universidades ibero-americanas no mesmo. A análise descritiva de aspectos relacionados à metodologia do Ranking THE permite a Ordorika e Gomez (2010) identificar limitações tais como: elevado grau de subjetividade das informações relacionadas à reputação; e predomínio de artigos em língua inglesa e de determinadas áreas do conhecimento em detrimento de outras para a composição dos indicadores de natureza objetiva.

Essas limitações metodológicas apontadas por Ordorika e Gomez (2010) são apontadas também por Valmorbida, Cardoso e Ensslin (2015) e Valmorbida et al. (2016) em trabalhos de natureza descritiva que identificam e analisam comparativamente os indicadores utilizados em diferentes rankings internacionais.

Théry (2010) realiza uma cartografia dos principais rankings universitários internacionais. Tomando como base as classificações de universidades a partir de quatro 
tradicionais rankings mundiais (ARWU; Ranking da École dês Mines de Paris - Mines ParisTech; Ranking THE; e Webometrics Ranking), o autor transfere seus resultados sobre um mapa-mundi e verifica que, de modo muito semelhante, cada um dos rankings concentra as "boas" ou "melhores" universidades em três regiões claramente definidas no globo: os Estados Unidos, a Europa e o Japão.

Aguillo e Granadino (2006) publicaram estudo empírico tratando especificamente do Webometrics Ranking, cujo propósito fundamental é medir o impacto e qualidade das universidades mundiais através de sua presença no universo cibernético. Os autores constatam a presença dominante de universidades norte-americanas nas primeiras posições desse ranking. O estudo suscita a hipótese de que se esteja criando uma "brecha digital", que pode levar, no futuro, à formas de colonialismo científico e cultural.

Bernardino e Marques (2010) examinam comparativamente os rankings THE, ARWU e CHE - ranking produzido pelo The German Centre for Higher Education Development - e propõem critérios a partir dos quais um ranking português poderia ser construído, tais como: evitar parâmetros e indicadores subjetivos; assegurar que os insumos relacionados à produção científica sejam tratados proporcionalmente ao tamanho das instituições; introduzir indicadores que permitam avaliar a qualidade do ensino, o grau de internacionalização da instituição e o percentual de financiamento oriundo de fontes privadas; entre outros.

A aplicabilidade dos rankings internacionais para a análise da realidade da educação de países latino-americanos é discutida por Vizcaino e de la Vega (2013). Em seu estudo, após análise do desempenho de quatro universidades venezuelanas em quatro rankings internacionais, os autores constatam a grande brecha em matéria de pesquisa e desenvolvimento existente entre os países centrais e os países latino-americanos periféricos, como a Venezuela.

Santos e Noronha (2016) realizam trabalho com o objetivo de analisar o desempenho das universidades brasileiras em três dos principais rankings internacionais, o ARWU, o THE e o QS em suas mais recentes edições. A conclusão dessa análise aponta para um desempenho bastante tímido das universidades brasileiras quando aferidas por meio das metodologias empregadas pelos principais rankings internacionais.

Finalmente, González-Riaño, Repiso e López-Cózar (2014) elaboram um estudo descritivo longitudinal retrospectivo sobre as notícias produzidas sobre rankings universitários na imprensa espanhola durante o período de 1998 a 2012. Constataram um incremento significativo de notícias a partir do ano de 2007, destacando dentre os rankings mais noticiados o ARWU, o SIR, o THE e o Webometrics. A conclusão dos autores aponta diretamente para o crescente destaque conferido pela mídia a respeito do fenômeno dos rankings e infere que, 
indiretamente, este destaque reflita o interesse de membros da comunidade acadêmica e científica, assim como dos gestores e responsáveis pelas políticas universitárias por informações sobre o impacto e o nível de seu próprio trabalho.

\section{b) A respeito de rankings regionais}

Foram identificados oito estudos sobre rankings regionais na literatura acadêmica educacional ibero-americana, procedente de autores vinculados a universidades de Cuba, Colômbia, Sérvia, Brasil e Espanha. O SIR - SCImago Institutions Rankings é o ranking regional que tem merecido maior atenção dos pesquisadores ibero-americano, o mesmo que utiliza como principal critério de avaliação da qualidade das universidades a sua capacidade de produzir e difundir pesquisas científicas. Os trabalhos de Dorta Contreras, Hernandez Ferraras e Cardenas-de-Baños (2011) e de Cardenas-de-Baños et al. (2012) tratam especificamente da posição que a Universidad de Ciencias Médicas de Habana ocupa no SIR Ranking, utilizando dados de 2010 e 2011, respectivamente, e defendem claramente a importância dessa escala como fonte de melhoria do prestígio local e internacional da IES. Trabalho bastante semelhante, analisando a realidade cubana, foi desenvolvido por Dorta Contreras e Rodriguez Rabelo (2011) ao analisar a inclusão da Universidad de Ciencias Informáticas de Cuba no SIR Ranking 2010.

O SIR Ranking também é utilizado por Mattar, González e Salgado (2013) como parâmetro de avaliação de universidades latino-americanas ao analisarem a situação das universidades colombianas a partir da classificação das mesmas nas edições 2010, 2011 e 2012 do SIR. Apesar das limitações metodológicas, para esses autores os rankings vêm se convertendo em ferramentas utilizadas pelas universidades para aumentar seu grau de visibilidade e competitividade. Outro estudo comparativo de universidades colombianas, tendo como parâmetro o SIR de 2010, é o artigo de Escobar-Córdoba, Toro-Herrera e EslavaSchmalbach (2010), que analisa a posição das escolas de medicina colombianas.

Os estudos de referencial empírico sobre o SIR envolvem não apenas análise do posicionamento de determinadas universidades no ranking em questão, identificou-se também estudos sobre seus aspectos metodológicos, dentre os quais Jeremic et al. (2013), que propõem a introdução de procedimentos estatísticos que permitam melhorar o grau de objetividade dos resultados expressos pelo SIR, e Lourenço e Calderón (2015) que efetuam um levantamento mais amplo de um grande conjunto de rankings regionais (dentre os quais o próprio $S I R$, o $Q S$ e o Webometrics) e nacionais que se espalham e proliferam no espaço ibero-americano.

Foi possível identificar também, na literatura científica examinada, ensaios sobre propostas de ranqueamento. Aguillo, Granadino e Llamas (2005) desenvolvem um estudo 
empírico com o objetivo de avaliar as universidades com base em sua presença e visibilidade na web, desenvolvendo uma proposta de ranking em boa medida semelhante ao Webometrics Ranking. Os autores comparam o resultado de seu ranking cibermétrico com os resultados do ARWU, identificando grande correlação entre as principais universidades latinoamericanas presentes nos dois rankings.

\section{c) A respeito de rankings nacionais}

Foi possível identificar doze estudos empíricos que tratam de rankings nacionais de dois países em particular: o Brasil e a Espanha. O primeiro artigo dessa natureza remonta ao ano de 1995. Partindo da constatação de que o Brasil não havia nenhum sistema de classificação de suas universidades que permitisse ordená-las segundo sua qualidade, Schwartzman (1995) propõe a construção de um ranking das universidades brasileiras, com base em três indicadores: o IQCD - Índice de Qualificação do Corpo Docente - utilizado como variável para aferir a produção acadêmica; a avaliação de Cursos de Graduação realizada à época por meio do Guia do Estudante, publicado pela Editora Abril; e a avaliação da pós-graduação, efetuada pela CAPES - Coordenadoria de Aperfeiçoamento de Pessoal da Educação Superior do Brasil. Em novo artigo, Schwartzman (1996) constrói um instrumento que avaliasse não as universidades, mas cursos de graduação de diferentes universidades. Nesse sentido, apresenta o Ranking IPEAD/UFMG ${ }^{3}$, testado em todos os cursos superiores de graduação em Administração, Economia e Ciências Contábeis do Estado de Minas Gerais, que adota indicadores sobre corpo docente; corpo discente; infraestrutura; e variáveis de resultado. Um ano após a publicação do artigo anteriormente mencionado, Schwartzman e Oliveira Junior (1997) publicam o resultado de uma pesquisa comparativa entre os resultados obtidos por meio do Ranking IPAD/UFMG e os resultados do primeiro ENC, indicando correlação positiva entre essas duas classificações.

A respeito dos rankings brasileiros, Andrade (2011) destaca em particular ações governamentais, como a implantação do Exame Nacional do Ensino Médio (ENEM) e do ENADE, bem como menciona rankings produzidos pelo mercado editorial, como é avaliação realizada pelo Guia do Estudante, que publica anualmente um ranking dos cursos de graduação no Brasil. Andrade (2011) propõe o exame dos diferentes tipos de rankings existentes, suas

\footnotetext{
${ }^{3}$ As siglas referem-se ao Instituto de Pesquisas Econômicas, Administrativas e Contábeis de Minas Gerais (IPAD) e à Universidade Federal de Minas Gerais (UFMG).
} 
características e os problemas existentes em cada um deles, de modo a melhor interpretar seus resultados e, eventualmente, propor mudanças que possam vir a aprimorá-los.

Embora Andrade (2011) mencione a existência de rankings brasileiros produzidos pelo mercado editorial, somente em 2014 pode-se identificar o primeiro estudo empírico sobre os mesmos. Trata-se do estudo de Calderón, Matias e Lourenço (2014), que aborda o extinto ranking Melhores Faculdades, editado anualmente pela Revista Playboy no período de 1982 a 2000. O artigo descreve o funcionamento, a metodologia utilizada e a evolução desse pioneiro ranking privado, utilizado para classificar cursos de graduação brasileiros de diversas áreas do conhecimento.

Ainda sobre os rankings brasileiros, Bittencourt, Casartelli e Rodrigues (2009) se debruçam no estudo do IGC, indicador oficial que busca expressar a qualidade global das universidades, a partir da avaliação de todos os seus Cursos de Graduação e de Pós-Graduação. Os autores analisam o desempenho das IES brasileiras a partir da divulgação dos primeiros resultados do IGC 2008, além de apresentar simulações da decomposição desse indicador de modo a auxiliar sua melhor interpretação e compreensão.

Os estudos empíricos relacionados a rankings nacionais espanhóis estão relacionados a duas iniciativas: o Observatório IUNE, criado com o objetivo de realizar análises sobre as atividades de pesquisa das universidades espanholas a partir de seis dimensões diferentes, a partir de quarenta e dois indicadores distintos, apresentados em artigo de Sanz-Casado et al. (2013), e o Ranking de Produtividade em Pesquisa das Universidades Públicas Espanholas, produzido por um grupo de pesquisadores espanhóis, que teve seus primeiros resultados divulgados no ano de 2009. A respeito desse segundo ranking, no primeiro de uma série de artigos divulgados por Buela-Casal et al. (2011) destaca-se que embora existam rankings exclusivamente voltados para a comparabilidade de universidades espanholas, caso do produzido e divulgado pelo diário $E l$ Mundo, e o $S I R$, por exemplo, nenhum deles é suficientemente capaz de avaliar as universidades espanholas a partir de sua efetiva produção científica e de acordo com as normas propostas pelos Princípios de Berlim para Rankings de Instituições de Educação Superior. Destaca-se a importância do dos rankings com vistas ao planejamento estratégico das universidades, uma vez que permite que cada uma delas se compare às demais, buscando identificar suas fragilidades e potencialidades e, por conseguinte, propor e implantar ações de melhoria, particularmente no que se refere à produção científica institucionalizada. Em quatro trabalhos posteriores Buela-Casal et al. $(2011 ; 2012 ; 2014)$ e Buela-Casal; Quevedo-Blasco e Ghuillén-Riquelme (2015) objetivam atualizar o ranking acima mencionado, renomeado de Ranking de Produção e Produtividade em Pesquisa das 
Universidades Públicas Espanholas, tomando como parâmetro dados coletados nos anos de 2010, 2011, 2012 e 2013, respectivamente, e obedecendo ao mesmo modelo de sua edição inicial, com pequenas nuances. Em conjunto, estes estudos apontam como principal problema da universidade espanhola a sua pouca visibilidade e competitividade frente ao novo contexto desencadeado a partir da criação do Espaço Europeu de Educação Superior.

\section{Considerações finais}

Calderón, Pfister e França (2015) haviam identificado, na literatura educacional brasileira, a emergência de um campo de estudos específico sobre os rankings a partir do início dos anos 2000. O presente estudo não apenas confirma esta hipótese, a partir do levantamento realizado junto a importantes bases de dados da literatura ibero-americana, mas aponta também para um processo de ampliação desse campo de estudos como objeto de interesse da comunidade científica educacional ibero-americana. $\mathrm{O}$ estudo evidenciou que a preocupação teórica pela compreensão dos rankings acadêmicos se concentra entre pesquisadores procedentes de instituições do Brasil (25 artigos), seguido por Espanha (11 artigos), México (cinco artigos), Cuba (três artigos) e Colômbia (dois artigos). Havendo também a curiosidade científica de pesquisadores procedentes de instituições de Portugal, Argentina, Chile e Honduras (um artigo de instituições de cada um desses países). Incluindo também pesquisadores de instituições fora do espaço ibero-americano: Sérvia, Estados Unidos e França (também um artigo por instituição de cada país).

A análise do estado da questão na literatura ibero-americana sobre a temática dos rankings acadêmicos revelou o caráter polissêmico dessa expressão, manifesto sobretudo, na profusão de sentidos que os diversos autores que tratam do tema emprestam ao mesmo. $\mathrm{O}$ levantamento efetuado permitiu demonstrar que a proliferação na produção de rankings globais, regionais e nacionais, começa a se fazer acompanhar também por uma maior atenção dos estudiosos da educação superior, como demonstrado na evolução da produção científica a respeito do assunto: dos 57 estudos identificados, três foram publicados na década de noventa do século passado, 18 no período compreendido entre os anos 2000 e 2009; e 36 apenas nos primeiros seis anos da presente década.

No período compreendido entre os anos 2000 e 2009 - época em que surgem os primeiros rankings globais, como o ARWU em 2003, e Webometrics e o THE em 2004; e no Brasil são criados os índices governamentais CPC e IGC, que viabilizam formas de ranqueamento oficial de cursos e universidades - prevalecem artigos teóricos-conceituais, 
críticos às políticas de ranqueamento nos sistemas educacionais, enquadrados no paradigma do conflito, que tendem a identificar o fenômeno dos rankings a processos de mercantilização da educação; à implantação de uma lógica racional produtivista típica das empresas privadas no seio das universidades; à difusão do modelo de "universidade de classe mundial" como paradigma de qualidade; enfatizando aspectos questionadores aos efeitos do modelo de universidade em desenvolvimento.

O estudo evidencia que a partir de 2010, prevalecem estudos teóricos conceituais mais alinhados ao paradigma do consenso, que tendem a enxergar o fenômeno do ranqueamento como um fator indutor de melhoria das instituições e sistemas de educação superior; de promoção de maior transparência na relação da oferta dos serviços educacionais; de seu papel enquanto fonte de informação para futuros estudantes; etc. É também a partir desse ano que se intensifica a produção de estudos de natureza empírica: 22 dos 29 artigos dessa natureza são desse período, evidenciando-se a preocupação por compreender melhor, de modo mais objetivo, aspectos relacionados à metodologia utilizada para a construção dos indicadores, de realizar estudos comparativos entre diferentes rankings e/ou sobre o desempenho de diferentes universidades de um país a partir de determinados rankings. Esses elementos parecem apontar uma tendência direcionada para o estudo sobre a performatividade institucional e de países nos rankings, compreendendo suas limitações, mas também suas potencialidades sem abrir mão de um exame mais cuidadoso e desapaixonado que procura elucidar as várias dimensões existentes nos processos de ranqueamento que, por sua natureza, são complexos, controversos e polissêmicos.

Finalmente, diante as tendências apontadas, será preciso acompanhar a produção científica sobre essa temática, ao longo dos próximos anos, a fim de examinar se efetivamente estamos diante de uma efetiva quebra de resistência em relação aos rankings e aos processos de ranqueamento e a uma elevação nos níveis de adesão a este procedimento como ferramenta e mecanismo de avaliação das universidades, dos cursos e dos sistemas de educação superior.

\section{Referências}

ALTBACH, P. International higher education: reflections on policy and practice. Chestnut Hill: Center for International Higher Education, Boston College, 2006.

AGUILLO, I. F.; GRANADINO, B. Indicadores web para medir la presencia de las universidades en la Red. RU\&SC. Revista de Universidad y Sociedad del Conocimiento, Barcelona, Espanha, v. 3, n. 1, p. 68-75, 2006. 
AGUILLO, I. F.; GRANADINO, B.; LLAMAS, G. Posicionamiento en el web del sector académico iberoamericano. Interciencia: Revista de Ciencia y Tecnologia de America, Caracas, Venezuela, v. 30, n. 12, p. 735-738, 2005.

ANDRADE, E. de C. Rankings em educação: tipos, problemas, informações e mudanças. Estudos Econômicos, São Paulo, v. 41, n. 2, p. 323-343, 2011.

ANZALDO, A. A. La universidad pública mexicana: el mito retórico frente a la realidad concreta. Ciencia Ergo Sum, Toluca, v. 11, n. 2, p. 16-25, 2004.

BARREYRO, G. B. Evaluación de la educación superior brasileña: el SINAES. Revista de la Educación Superior, Distrito Federal, México, v. 35, n. 137, p. 63-73, 2006.

BARREYRO, G. B. De exames, rankings e mídia. Avaliação, Campinas; Sorocaba, v. 13, n. 3, p. 863-868, 2008.

BARSKY, O. Acerca de los rankings internacionales de las universidades y su repercusión en Argentina. Revista Debate Universitario, Buenos Aires, v. 1, n. 1, p. 30-78, 2012.

BERNARDINO, P.; MARQUES, R. C. Academic rankings: an approach to rank portuguese universities. Ensaio, Rio de Janeiro, v. 18, n. 66, p. 29-48, 2010.

BITTENCOURT, H. R.; CASARTELLI, A. de O.; RODRIGUES, A. C. de M. Sobre o índice geral de cursos (IGC). Avaliação, Campinas; Sorocaba, v. 14, n. 3, p. 667-682, 2009.

BRITO, M. R. F. de. O SINAES e o ENADE: da concepção à implantação. Avaliação, Campinas; Sorocaba, v. 13, n. 3, p. 841-850, 2008.

BUELA-CASAL, G. et al. Ranking de 2010 en producción y productividad en investigación de las universidades públicas españolas. Psicothema, Oviedo, v. 23, n. 4, p. 527-536, 2011.

BUELA-CASAL, G. et al. Ranking de 2011 en producción y productividad en investigación de las universidades públicas españolas. Psicothema, Oviedo, v. 24, n. 4, p. 505-515, 2012.

BUELA-CASAL, G. et al. Ranking 2012 de investigación de las universidades públicas españolas. Psicothema, Oviedo, Espanha, v. 26, n. 2, p. 149-158, 2014.

BUELA-CASAL, G.; QUEVEDO-BLASCO, R.; GUILLÉN-RIQUELME, A. Ranking 2013 de investigación de las universidades públicas españolas. Psicothema, Oviedo, v. 27, n. 4, p. 317-326, 2015.

CALDERON PADILLA, R. El crecimiento y desarrollo de la educación superior en Honduras, una perspectiva desde la UNAH. Revista Innovación Educativa, Distrito Federal, México, v. 11, n. 57, p. 81-89, 2011.

CALDERÓN, A. I.; PFISTER, M.; FRANÇA, C. M. Rankings acadêmicos na educação superior brasileira: a emergência de um campo de estudo (1995-2013). Roteiro, Joaçaba, Santa Catarina, v. 40, n. 1, p. 31-50, 2015.

CALDERÓN, A. I.; LOURENÇO, H. da S. Rankings en la educación superior brasileña: una aproximación a los rankings públicos y privados. Revista Digital de Investigación en Docencia Universitaria, Lima, Peru, v. 8, n. 1, p. 95-110, 2014.

CALDERÓN, A. I.; MATIAS, R. C.; LOURENÇO, H. da S. Rankings na educação superior: as melhores faculdades do Brasil (1982-2000). Estudos em Avaliação Educacional, São Paulo, v. 25, n. 57, p. 226-247, jan./abr. 2014.

CALDERÓN, A. I.; POLTRONIERI, H.; BORGES, R. M. Os rankings na educação superior brasileira: políticas de governo ou de estado? Ensaio, Rio de Janeiro, v. 19, n. 73, 2011. 
CARDENAS-DE-BAÑOS, L. et al. La productividad de la ciencia en la Universidad de Ciencias Médicas de La Habana y los desafíos a los mecanismos de medición del conocimiento. Revista Cubana de Información en Ciencias de la Salud, Havana, v. 23, n. 4, p. 391-403, 2012.

CHARLOT, B.; SILVA, V. A. da. De Abelardo até a classificação de Xangai: as universidades e a formação dos docentes. Educar em Revista, Curitiba, n. 37, p. 39-58, 2010.

CHIZZOTTI, A.; PONCE, B. J. Avaliação da produção docente no ensino superior: possibilidades e limites. Revista e-Curriculum, São Paulo, v. 5, n. 2, p. 1-19, 2010.

DORTA CONTRERAS, A. J.; HERNANDEZ FERRERAS, K.; CARDENAS-DE-BAÑOS, L. Calidad de la ciencia producida en la Universidad de Ciencias Médicas de La Habana: un modelo y nuevos retos. Revista Habanera de Ciencias Médicas, Havana, v. 10, n. 1, p. 99$101,2011$.

DORTA CONTRERAS, A. J.; RODRIGUEZ RABELO, A. Producción científica en la Universidad de las Ciencias Informáticas. RCIM - Revista Cubana de Informática Médica, Havana, v. 3, n. 1, p. 64-74, janeiro-junho, 2011.

ESCOBAR-CÓRDOBA, F.; TORO-HERRERA, S. M.; ESLAVA-SCHMALBACH, J. Posición de las Escuelas de Medicina colombianas a partir del Ranking Iberoamericano SIR2010. Revista Facultad de Medicina de la Universidad Nacional de Colombia, Bogotá, v. 58, n. 4, p. 341-347, 2010.

GOMES, C. A. A educação em novas perspectivas sociológicas. São Paulo: EPU, 2005.

GONÇALVES, A.; CALDERON, A. I. Academic rankings in higher education: trends of international scientific literature. Revista Diálogo Educacional, v. 17, p. 1125-1145, 2017.

GONZÁLEZ-RIAÑO, M. G.; REPISO, R.; LÓPEZ-CÓZAR, E. D. Repercusión de los rankings universitarios en la prensa española. Revista Española de Documentación Científica, Madrid, v. 37, n.3, p. 1-9, 2014.

HAZELKORN, E. Globalization and the reputation race in rankings and the reshaping of higher education: the battle for world class excellence. London: Palgrave MacMillan, 2011.

JEREMIC, V. et al. Excelencia con liderazgo: El indicador rey del Scimago Institutions Rankings Iber Report. El profesional de la información, Barcelona, v. 22, n. 5, p. 474-479, 2013.

LEITE, D. Ameaças pós-rankings sobrevivência das CPAS e da auto-avaliação. Avaliação, Campinas; Sorocaba, v. 13, n. 3, p. 833-840, 2008.

LOPEZ SEGRERA, F. Tendencias de la educación superior en el mundo y en América Latina y el Caribe. Avaliação, Campinas; Sorocaba, v. 13, n. 2, p. 267-29, 2008.

LOPEZ SEGRERA, F. Reflexiones en torno a la financiación actual y futura de las universidades a nivel mundial. Avaliação, Campinas; Sorocaba, v. 14, n. 3, p. 523-560, 2009.

LÓPEZ SEGRERA, F. Educación superior comparada: tendencias mundiales y de América Latina y Caribe. Avaliação, Campinas; Sorocaba, v. 21, n. 1, p. 13-32, 2016.

LOURENÇO, H. da S.; CALDERÓN, A. I. Rankings acadêmicos na educação superior: mapeamento da sua expansão no espaço ibero-americano. Acta Scientiarum: Education, Maringá, v. 37, n. 2, p. 187-197, 2015. 
MARGINSON, S. University Rankings and Social Science. European Journal of Education, v. 49, n. 1, p. 45-59, 2014.

MARTINS, C. B. Notas sobre a formação de um sistema transnacional de ensino superior. Caderno CRH, Salvador, v. 28, n. 74, p. 291-308, 2015.

MATTAR, S. V.; GONZÁLEZ, M. T.; SALGADO, L. A. Análisis de las universidades colombianas de acuerdo con el ranking SCImago 2010-2012. Revista MVZ Córdoba, Córdoba, v. 18, n. 1, p. 3399-3407, 2013.

MENEGHEL, S. M.; ROBL, F.; FREITAS DA SILVA, T. T. A relação entre avaliação e regulação na educação superior: elementos para o debate. Educar em Revista, Curitiba, n. 28, p. 89-106, 2006.

MOURA, B. A; MOURA, L. B. A. M. Ranqueamento de universidades: reflexões acerca da construção de reconhecimento institucional. Acta Scientiarum: Education, Maringá, v. 35, n. 2, p. 213-222, 2013

NÓBREGA-THERRIEN, S. M.; THERRIEN, J. Trabalhos científicos e o estado da questão: reflexões teórico-metodológicas. Estudos em Avaliação Educacional, São Paulo, v. 15, n. 30, p. 5-16, 2004.

ORDORIKA SACRISTÁN, I. Educación superior y globalización: las universidades públicas frente a una nueva hegemonía. Andamios. Revista de Investigación Social, Distrito Federal, México, v. 3, n. 5, p. 31-47, 2006.

ORDORIKA, I.; RODRIGUEZ GOMEZ, R. El ranking Times en el mercado del prestigio universitario. Perfiles Educativos, Distrito Federal, México, v. 32, n. 129, janeiro, 2010.

REYES, C. Medición de la calidad universitária en Chile: la influencia de los rankings. Calidad en la Educación, Santiago, n. 44, p. 158-196, 2016.

RODRÍGUEZ SABIOTE, C.; GUTIÉRREZ PÉREZ, J. Debilidades de la evaluación de la calidad en la universidad española. Causas, consecuencias y propuestas de mejora. REDIE. Revista Electrónica de Investigación Educativa, Ensenada, v. 5, n. 1, p. 1-16, 2003.

ROTHEN, J. C.; SCHULZ, A. SINAES: do documento original à legislação. Revista Diálogo Educacional, Curitiba, v. 7, n. 21, p. 163-180, 2007.

SANTOS, S. M. dos; NORONHA, D. P. O desempenho das universidades brasileiras em rankings internacionais. Em Questão, Porto Alegre, v. 22, n. 2, p. 186-219, 2016.

SANZ-CASADO, E. et al. Rankings nacionales elaborados a partir de múltiples indicadores frente a los de índices sintéticos. Revista Española de Documentación

Científica, Madrid, v. 36, n. 3, p. 1-18, 2013.

SALMI, J. The challenge of establishing world-class universities. Washington DC: The International Bank for Reconstruction and Development / The World Bank, 2009.

SCHWARTZMAN, J. Dificuldades e possibilidades de se construir um ranking para as universidades brasileiras. Ensaio, Rio de Janeiro, v. 3, n. 6, p. 5-28, 1995.

SCHWARTZMAN, J. Uma metodologia de avaliação de cursos de graduação. Ensaio, Rio de Janeiro, v. 4, n. 12, p. 237-264, 1996.

SCHWARTZMAN, J.; OLIVEIRA JÚNIOR, M. de. "Ranking” IPEAD/UFMG e Exame Nacional de Cursos: uma comparação. Ensaio: Avaliação e Políticas Públicas em

Educação, Rio de Janeiro, v. 5, n.16, p. 353-362, 1997. 
SEOANE, A. Y. Como se evalúan las universidades de clase mundial? Revista de la Educación Superior, Distrito Federal, México, v. 38, n. 150, p. 113-120, 2009.

SEOANE, M. J. F.; ÁLVAREZ, M. T. Revisión histórica de la garantía de calidad externa en las instituciones de educación superior. Revista de la Educación Superior, Distrito Federal, México, v. 39, n. 155, p. 123-135, 2010.

SGUISSARDI, V. Regulação estatal versus cultura de avaliação institucional? Avaliação, Campinas; Sorocaba, v. 13, n. 3, p. 857-862, 2008.

TELLES SCAGLIONE, V. L.; PITPAR GOLDCHLEGER, L. A escolha de uma universidade vista sob o prisma da teoria do comportamento do consumidor. Revista Gestão Universitária na América Latina - GUAL, Florianópolis, v. 9, n. 4, p. 92-108, 2016.

THÉRY, H. Classificações de universidades mundiais, "Xangai" e outras. Estudos Avançados, São Paulo, v. 24, n. 70, p. 185-205, 2010.

VALMORBIDA, S. M. I.; CARDOSO, T. L.; ENSSLIN, S. R. Rankings universitários: análise dos indicadores utilizados. Sociedade, Contabilidade e Gestão, Rio de Janeiro, v. 10, n. 2, p. 88-102, 2015.

VALMORBIDA, S. M. I. et al. Rankings universitários mundiais. Que dizem os estudos internacionais? REICE. Revista Iberoamericana sobre Calidad, Eficacia y Cambio en Educación, Madrid, v. 14, n. 2, p. 5-29, 2016.

VILLASEÑOR BECERRA, J. I.; MORENO ARELLANO, C. I.; FLORES OROZCO, J. E. Perspectivas actuales sobre los rankings mundiales de universidades. Revista de la Educación Superior, Distrito Federal, México v. 44, n. 175, p. 41-67, 2015.

VIZCAINO, G.; DE LA VEGA, I. Rankings de universidades ¿aplican para países periféricos como Venezuela? Interciencia, Caracas, Venezuela, v. 38, n. 10, p. 745-752, out. 2013.

ZAINKO, M. A. S. Avaliação da educação superior no Brasil: processo de construção histórica. Avaliação, Campinas; Sorocaba, v. 13, n. 3, p. 827-831, nov. 2008.

Adolfo Ignacio Calderón ${ }^{1}$ Pontifícia Universidade Católica de Campinas | Programa de Pós-Graduação em Educação Campinas | SP | Brasil. Contato: adolfo.ignacio@puc-campinas.edu.br ORCID (iD) http://orcid.org/0000-0001-6534-2819

Carlos Marshal França ${ }^{2}$ Pontifícia Universidade Católica de Campinas | Faculdade de Administração do Centro de Economia e Administração Campinas | SP | Brasil. Contato: carlos.marshal@ @uc-campinas.edu.br ORCID (iD) http://orcid.org/0000-0002-4039-1689 